\title{
A GENERALIZED KOSZUL COMPLEX
}

\author{
BY DAVID A. BUCHSBAUM AND DOCK S. RIM ${ }^{1}$
}

Communicated by D. Zelinsky, December 20, 1962

1. This note is concerned with the definition of a free complex which generalizes the classical Koszul complex [2], and its application to the notions of depth and multiplicity.

Let $f: R^{m} \rightarrow R^{n}$ be a map, where $R$ is any commutative ring. Then for each $p, 1 \leqq p \leqq n$, we define a complex $K$ as follows:

$$
\begin{aligned}
K_{q+2} & =\sum \stackrel{s_{0}+\nu}{\Lambda} R^{n *} \otimes \Lambda^{s_{1}} R^{n *} \otimes \cdots \otimes \Lambda^{s_{q}} R^{n *} \otimes \stackrel{n+1+\Sigma_{s_{i}}}{\Lambda} R^{m} \quad \text { for } q \geqq 0 ; \\
K_{1} & =\bigwedge^{p} R^{m}, \quad K_{0}=\Lambda^{p} R^{n} ;
\end{aligned}
$$

$\nu$ is the fixed integer $n+1-p$ and the summation is taken over all $s_{0} \geqq 0, s_{i} \geqq 1$ for $i \geqq 1$. (We are using the notation $M^{*}=\operatorname{Hom}(M, R)$ for any $R$-module $M$.) The boundary map in this complex is defined as

$$
\begin{aligned}
d_{q+2}\left(b_{0}\right. & \left.\otimes b_{1} \otimes \cdots \otimes b_{q} \otimes a\right) \\
= & \sum_{i=0}^{q-1}(-1)^{i} b_{0} \otimes \cdots \otimes b_{i} \wedge b_{i+1} \otimes \cdots \otimes b_{q} \otimes a \\
& \quad+(-1)^{q} b_{0} \otimes \cdots \otimes b_{q-1} \otimes \omega_{b_{q}}(a) \quad \text { for } q \geqq 0,
\end{aligned}
$$

and $d_{1}: \Lambda^{p} R^{m} \rightarrow \Lambda^{p} R^{n}$ is simply $\Lambda^{p} f$. The symbol $\omega_{b_{q}}(a)$ is defined as follows: if $\beta$ is any element of $R^{n *}$, then $\beta f$ is in $R^{m *}$ and thus induces a derivation of degree -1 on the exterior algebra of $R^{m *}$, denoted by $\omega_{\beta}$. If $b=\beta_{1} \wedge \cdots \wedge \beta_{s} \in \Lambda^{\cdot} \cdot R^{n *}$ and $a \in \Lambda^{t} R^{m}$, then $\omega_{b}(a)$ is defined to be $\omega_{\beta_{1}} \cdots \omega_{\beta_{a}}(a)$. Since $\omega_{\beta_{1}} \omega_{\beta_{2}}+\omega_{\beta_{2}} \omega_{\beta_{1}}=0$, this operation is well defined and thus gives a pairing $\Lambda^{*} R^{n *} \otimes \Lambda^{t} R^{m} \rightarrow \Lambda^{t-s} R^{m}$.

The fact that this gives a chain complex is rather easy to verify and the length of the complex is seen to be $m-n+1$.

2. If $R$ is a commutative, noetherian ring, and $E$ is an $R$-module, a sequence $a_{1}, \cdots, a_{d}$ of elements in $R$ is called a proper $E$-sequence if for all $i, 1 \leqq i \leqq d, E /\left(a_{1}, \cdots, a_{i}\right) E \neq 0$ and $a_{i}$ is not a zero-divisor for $E /\left(a_{1}, \cdots, a_{i-1}\right) E$ [1]. If $I$ is an ideal of $R$, the $I$-depth of $E$ is defined to be the length of a maximal proper $E$-sequence of elements contained in $I$. It is known that this number is always finite for a

1 This work was done with the partial support of NSF grant G-14097 and also with the partial support of IDA. 
noetherian ring $R$, and that any two such maximal proper $E$-sequences have the same length [1].

If $f: R^{m} \rightarrow R^{n}$, the ideal $I(f)$ is defined to be the annihilator of Coker $\Lambda^{n} f$. If $E$ is an $R$-module, we denote the homology of $K \otimes E$ by $H_{*}\left(\Lambda^{p} f, E\right)$, and that of $\operatorname{Hom}(K, E)$ by $H^{*}\left(\Lambda^{p} f, E\right)$.

TheOREM 1. Given a map $f: R^{m} \rightarrow R^{n}(m \geqq n$, and $R$ a noetherian ring) and an $R$-module $E$ such that $E / I(f) E \neq 0$, we have, for each $p(1 \leqq p \leqq n)$ the following statements:

(1) $I(f)$-depth $E$ is the smallest integer $q$ for which $H^{q}\left(\Lambda^{p} f, E\right) \neq 0$, and $H^{d}\left(\Lambda^{p} f, E\right)=\operatorname{Ext}^{d}\left(\right.$ Coker $\left.\Lambda^{p} f, E\right)$, where $d=I(f)$-depth $E$.

(2) $m-n+1-(I(f)$-depth $E)=$ the largest integer $q$ for which $H_{q}\left(\Lambda^{p} f, E\right) \neq 0$. Furthermore, $H_{m-n+1-d}\left(\Lambda^{p} f, E\right)$ may also be interpreted as $\operatorname{Ext}^{d}(M, E)$ where $M$ is a specific module depending upon $f$ and $p$.

The proof of this theorem follows from a fairly general argument about exact connected sequences of functors $\left\{T^{i}\right\}$ satisfying several conditions, the main one being that for every $R$-module $E$, Supp $T^{i}(E)$ $C R / I$ for some fixed ideal $I$. It can be shown that $H^{*}\left(\Lambda^{p} f, E\right)$ and $H_{*}\left(\wedge^{p} f, E\right)$ (the latter with suitable shift of index) are both exact connected sequences of functors of the appropriate type, and hence our result.

As a corollary, we obtain the fact that if $E$ and $f$ are as above, then $I(f)$-depth $E \leqq m-n+1$. We also obtain the generalized CohenMacaulay unmixedness theorem, due to Eagon [3] which is:

THEOREM 2. Let $f: R^{m} \rightarrow R^{n}(m \geqq n)$ be a map such that $I(f)$-depth $R=m-n+1$. Then if $R$ is a Cohen-Macaulay ring, Coker $\Lambda^{p} f$ is unmixed for all $p, i \leqq p \leqq n$.

The proof proceeds by using the fact that if $I(f)$-depth $R=m-n+1$, then $K_{\Lambda^{p}}$, is an acyclic resolution of Coker $\Lambda^{p} f$ and $K_{\Lambda^{p}}^{*}$ $=\operatorname{Hom}\left(K_{\Lambda^{p}}, R\right)$ is an acyclic resolution of $H^{m-n+1}\left(\Lambda^{p} f, R\right)$. Thus $\operatorname{Ext}^{i}\left(H^{m-n+1}\left(\Lambda^{p} f, R\right), R\right)$ is 0 if $i<m-n+1$, and is Coker $\Lambda^{p} f$ if $i=m-n+1$. Thus one may express Coker $\Lambda^{p} f$ as $\operatorname{Ext}^{m-n+1}\left(H^{m-n+1}\left(\bigwedge^{p} f, R\right), R\right)$ which is equidimensional. Since $R$ is Cohen-Macaulay, this implies that Coker $\Lambda^{p} f$ is unmixed.

3. Let us assume throughout that $R$ is a local ring, ${ }^{2}$ unless otherwise specified. Although this is not an essential assumption, it simplifies some of our statements.

If $M$ is an $R$-module, we denote by $S(M)=\sum S_{\nu}(M)$ the symmetric

2 Here, by a local ring, we mean a commutative, noetherian ring with identity element, having a unique maximal ideal. 
algebra generated by $M$ over $R$. If $f: R^{m} \rightarrow R^{n}$ is a map, then $S_{\nu}(f)$ : $S_{\nu}\left(R^{m}\right) \rightarrow S_{\nu}\left(R^{n}\right)$ is the induced map on symmetric products. If, moreover, $E$ is an $R$-module such that Coker $f \otimes E$ has finite length, then the same is true for Coker $S_{\nu}(f) \otimes E$ for every $\nu \geqq 1$, i.e., $l\left(\right.$ Coker $\left.S_{\nu}(f) \otimes E\right)<\infty$. We define a numerical function $P_{f}(\nu, E)$ $=l\left(\right.$ Coker $\left.S_{\nu}(f) \otimes E\right)$.

For any polynomial function $\phi$, we define $\mu(\phi)=(\operatorname{deg} \phi)$ ! (leading coefficient of $\phi$ ).

THEOREM 3. Let $R$ be a local ring, $f: R^{m} \rightarrow R^{n}$ be a map, and $E$ be an $R$-module such that $l($ Coker $f \otimes E)<\infty$. Then

(1) $P_{f}(\nu, E)$ is a polynomial function for all sufficiently large $\nu$;

(2) $\mu\left(P_{f}(\nu, E)\right)$ depends only on Coker $f$ and $E$;

(3) $\operatorname{deg} P_{f}(\nu, E)=n-1+\operatorname{dim} E$.

The proof, although not trivial, is computational. Let $\tau^{m}: S\left(R^{m}\right)^{m}$ $\rightarrow S\left(R^{m}\right)$ be the canonical map. $S\left(R^{n}\right)$, and hence also $S\left(R^{n}\right) \otimes E$, becomes a graded $S\left(R^{m}\right)$-module through the map $S(f): S\left(R^{m}\right) \rightarrow S\left(R^{n}\right)$. Hence we may consider the Koszul complex $K_{\tau^{m}}$ associated with the map $\tau^{m}$, and the (graded) homology groups $H_{*}\left(\tau^{m}, S\left(R^{n}\right) \otimes E\right)$ whose $\lambda$ th homogeneous part is denoted by $H_{*}\left(\tau^{m}, S\left(R^{n}\right) \otimes E\right)_{\lambda}$. We then observe that $\Delta^{m} P_{f}(\nu, E)=\sum(-1)^{m-q} l\left(H_{m-q}\left(\tau^{m}, S\left(R^{n}\right) \otimes E\right)_{\nu+q}\right)$ for all sufficiently large $\nu$, where $\Delta^{m} P_{f}$ denotes the $m$ th difference function of $P_{f}$, and establishes, through the use of a certain double complex, that $\Delta^{m+n} P_{f}(\nu, E)=0$ for all sufficiently large $\nu$. This establishes (1).

The proof of (2) is a purely formal computation, based on an application of Schanuel's lemma, which also shows that $\operatorname{deg} P_{f}(\nu, E)$ $-n+1$ depends only on Coker $f$ and $E$.

Having proved these facts, the proof of (3) proceeds by restricting our attention to maps $f: R^{m} \rightarrow R^{n}$ such that $f\left(R^{m}\right) \subset \mathfrak{m} R^{n}$, where $\mathfrak{m}$ is the maximal ideal of $R$. Since, then, $I(f) \subset \mathfrak{m}$, deg $P_{f}-n+1$ gets squeezed between the degree of the characteristic function for $\mathfrak{m}$ and that for $I(f)$ with respect to $E$. Hence the result.

As a consequence of this theorem, we obtain a generalization of Krull's principal ideal theorem.

THEOREM 4. Let $R$ be any noetherian ring, and $f: R^{m} \rightarrow R^{n}$, with $m \geqq n$. Then $\operatorname{dim} R_{\mathfrak{p}} \leqq m-n+1$ for all minimal primes $p$ in Supp Coker $f$.

The proof proceeds by first reducing to the case when $R$ is a local ring and Coker $f$ has finite length. One then may assume that $R$ is an integral domain and thus contains a nonzero divisor $\alpha$ in its maximal ideal. From the exact sequence $0 \rightarrow S\left(R^{n}\right) \rightarrow^{\alpha} S\left(R^{n}\right)$ $\rightarrow S\left(R^{n}\right) \otimes R / \alpha \rightarrow 0$ (the maps being of homogeneous degree 0 ), and 
using the fact that $\Delta^{m} P_{f}$ may be computed as an Euler-Poincaré characteristic, together with Theorem 3, we obtain our result.

We also obtain as a corollary (again $R$ is a local ring) that if $f: R^{m} \rightarrow R^{n}$ and $E$ is such that $l($ Coker $f \otimes E)<\infty$, then $m-n+1$ $\geqq \operatorname{dim} E$ and hence $m \geqq \operatorname{deg} P_{f}(\nu, E)$.

These theorems naturally lead to the following definition. Let $M$ be a module of finite length over a local ring $R$, let $f: R^{m} \rightarrow R^{n}$ be a map whose cokernel is $M$, and let $E$ be an $R$-module. Then ( $\operatorname{dim} R+n-1)$ ! (the coefficient of the term of degree $n-1+\operatorname{dim} R$ in the polynomial $P_{f}(\nu, E)$ ) is a non-negative integer which depends only on $M$ and $E$. We call it the multiplicity of $M$ with respect to $E$, and denote it by $e_{E}(M)$.

THEOREM 5. If $R$ is a local ring, given a map $f: R^{m} \rightarrow R^{n}$ and an $R$-module $E$ such that $l($ Coker $f \otimes E)<\infty$, we have

$$
\left(\begin{array}{l}
n-1 \\
n-p
\end{array}\right) \Delta^{m} P_{f}(\nu, E)=\chi H_{*}(\stackrel{p}{\Lambda} f, E)
$$

where $\chi H_{*}\left(\bigwedge^{p} f, E\right)=\sum_{q}(-1)^{q} l\left(H_{q}\left(\bigwedge^{p} f, E\right)\right)$.

The proof of this theorem falls out of a general construction of a double complex associated with maps $R^{m} \rightarrow^{f} R^{n} \rightarrow^{o} R^{r}$ which relates the complexes $K$ defined by $f, g$, and $g f$.

We say the map $f: R^{m} \rightarrow R^{n}$ is a parameter matrix for $R$ if $M=\operatorname{Coker} f$ has finite length, and $m-n+1=\operatorname{dim} R$. We obtain as a corollary of the above theorem that if $f: R^{m} \rightarrow R^{n}$ is a parameter matrix for $R$ with cokernel $M$, and if $E$ is an $R$-module, then

$$
\chi H_{*}(\stackrel{p}{\Lambda} f, E)=\left(\begin{array}{l}
n-1 \\
n-p
\end{array}\right) e_{E}(M)
$$

\section{BIBLIOGRAPHY}

1. M. Auslander and D. A. Buchsbaum, Homological dimension in noetherian rings. II, Trans. Amer. Math. Soc. 88 (1958), 194-206.

2. - Codimension and multiplicity, Ann. of Math.(2) 68 (1958), 625-657.

3. J. Eagon, Ideals generated by the subdeterminants of a matrix, $\mathrm{Ph} . \mathrm{D}$. dissertation, Univ. of Chicago, Chicago, Ill., 1961.

BRANDEIS UNIVERSITY 